\title{
A Combined Regular-Logarithmic Perturbation Method for Signal-Noise Interaction in Amplified Optical Systems
}

\author{
Marco Secondini, Enrico Forestieri, Member, IEEE, and Curtis R. Menyuk, Fellow, IEEE
}

\begin{abstract}
We present a novel perturbation method for the nonlinear Schrödinger equation (NLSE) that governs the propagation of light in optical fibers. We apply this method to study signal-noise interactions in amplified multispan fiber-optic systems. Being based on a combination of the regular perturbation (RP) and logarithmic perturbation, the method is especially suitable for modeling the simultaneous presence of nonlinear and dispersive effects. Even after linearization, it retains the contribution of the quadratic perturbation terms of the NLSE, thereby achieving higher accuracy than an RP with comparable complexity. We revise parametric gain and nonlinear phase-noise effects under the new theory. We finally consider several examples and evaluate the probability density function of the optical or postdetection signal and the bit-error rate of an NRZ-OOK system. All of the results are compared with other models and with multicanonical Monte Carlo simulations.
\end{abstract}

Index Terms-Communication system performance, KarhunenLoève transforms, Monte Carlo methods, nonlinearities, opticalfiber theory, optical Kerr effect, optical noise, parametric gain, perturbation methods, phase noise.

\section{INTRODUCTION}

$\mathbf{T}$ HE propagation of light in optical fibers is governed by the nonlinear Schrödinger equation (NLSE) and its variants [1]. For deterministic signals, the problem can be solved either by numerical methods [1], [2] or analytical approximations [3]-[7]. In this case, the choice of the method is a matter of accuracy and computational efficiency, which can be optimized by controlling parameters, such as the step size in the split-step Fourier method [2], or the recursion depth in the regular perturbation (RP) or logarithmic perturbation (LP) expansion [7]. On the other hand, special attention must be paid to the propagation of a stochastic process, such as an optical signal affected by the amplified spontaneous emission (ASE) noise generated by optical amplifiers to determine its output statistics. In systems operating in the linear regime, ASE noise is not affected by propagation through the optical fiber, and can be

Manuscript received September 01, 2008; revised December 17, 2008. Current version published July 24, 2009. This work was supported in part by Ericsson and in part by the MIUR PRIN project STORICO.

M. Secondini and E. Forestieri are with the Scuola Superiore Sant'Anna di Studi Universitari e di Perfezionamento, Pisa I-56124, Italy (e-mail: marco.secondini@sssup.it; forestieri@sssup.it).

C. R. Menyuk is with the University of Maryland, Baltimore County, Baltimore, MD 21250 USA (e-mail: menyuk@umbc.edu).

Color versions of one or more of the figures in this paper are available online at http://ieeexplore.ieee.org.

Digital Object Identifier 10.1109/JLT.2009.2012873 modeled as additive white Gaussian noise at the end of the link. On the other hand, when nonlinear effects are not negligible, signal and noise interactions occur during propagation. As a result, at certain frequencies, the noise may be amplified or attenuated, and become colored. This effect is called parametric gain. Additionally, the probability distribution function for the noise samples may change, becoming nonstationary and even non-Gaussian. In previous work [3], [8], [9], parametric gain has been studied by an RP expansion of the nonlinear Schrödinger equation (NLSE). For a small perturbation, only the first-order terms of the RP can be retained, and the NLSE can be linearized. More recently, other work has shown that a simple linearization does not hold in many cases [10] and second-order terms may not be neglected, since they can produce a strong degradation of the system performance [11]. In the covariance matrix method [10], the RP expansion is applied to a modulated signal, and phase jitter is extracted (eliminated) from each pulse during propagation in order to maintain the validity of the linearization hypothesis. In this paper, we present a novel perturbation approach [12] to the NLSE, derived by a combination of the RP and LP expansions described in [7]. The intuitive idea behind this approach, such as in [7] and [10], is that the nonlinearity produces phase rotations and so at high powers, one wishes to perturb the phase and amplitude (LP approach), rather than the two quadratures (RP approach) of the signal. The difficulty when perturbing the amplitude and phase is to avoid singularities at low power, and our approach does that. Moreover, if we try to map the Gaussian noise introduced by amplifiers onto an amplitude-phase basis, its components are no longer Gaussian distributed. Our approach also avoids this problem. We derive a set of differential equations for the propagation of the perturbed solution of the NLSE. As in the RP approach, the equations can be linearized. The resulting linear model is simple, but, being based on a more convenient basis, it is more robust than the linearized RP model. The model gives a complete statistical characterization of the received optical signal and is suitable for the evaluation of system performance.

This paper is organized as follows. In Section II, we introduce the combined regular-logarithmic perturbation (CRLP) model and derive the basic propagation equations. In Section III, we apply the model to the propagation of ASE noise in amplified multispan optical systems. In Section IV, we consider some simple systems to discuss the characteristics and accuracy of the proposed model and compare it with other models. Section V shows some examples, where we evaluate the probability density function (pdf) of the optical and postdetection signal, and 
the bit-error rate (BER) of an NRZ-OOK system, and compare the results obtained by the CRLP model with those obtained by other models and by simulations. Finally, in Section VI, we discuss the results and give the conclusions.

\section{Combined Regular-Logarithmic PERTURBATION (CRLP)}

In general, the propagation of light in optical fibers is governed by the NLSE and its variants [1]. Neglecting polarization effects and accounting for chromatic dispersion, self-phase modulation, and loss, in a time frame moving with the signal group velocity, the NLSE can be written as

$$
\frac{\partial v}{\partial z}=j \frac{\beta_{2}}{2} \frac{\partial^{2} v}{\partial t^{2}}-j \gamma|v|^{2} v-\frac{\alpha}{2} v
$$

where $v(z, t)$ is the optical field complex envelope, $\alpha$ is the power attenuation constant, $\beta_{2}$ is the chromatic dispersion coefficient, and $\gamma$ is the Kerr nonlinear coefficient. By defining $v(z, t) \triangleq \exp (-\alpha z / 2) u(z, t)$, we can eliminate the last term in (1), which becomes

$$
\frac{\partial u}{\partial z}=j \frac{\beta_{2}}{2} \frac{\partial^{2} u}{\partial t^{2}}-j \gamma \exp (-\alpha z)|u|^{2} u .
$$

As performed in [3], [8], and [9], we make the hypothesis that for the purpose of investigating the interaction between signal and noise, the signal can be considered a continuous wave (CW), and the noise can be treated as a perturbation of the $\mathrm{CW}$ solution of the NLSE. The novelty of our approach is that instead of the classical RP expansion

$$
u_{\mathrm{RP}}(z, t)=\sqrt{P_{0}}\left[1+c^{\prime}(z, t)\right] \exp \left[-j \phi_{\mathrm{NL}}(z)\right]
$$

where $P_{0}$ is the power of the noise-free solution $c^{\prime}(z, t)=$ $a^{\prime}(z, t)+j b^{\prime}(z, t)$ is the additive perturbation, and

$$
\phi_{\mathrm{NL}}(z)=\int_{0}^{z} \gamma P_{0} \exp (-\alpha \xi) d \xi
$$

is the deterministc time-independent nonlinear phase rotation of the noise-free solution, we write the perturbed solution of the NLSE as being

$$
u(z, t)=\sqrt{P_{0}}[1+c(z, t)] \exp [-j \theta(z, t)] .
$$

Equation (5) is motivated by the evidence, given in [7], that the LP solution of the NLSE can be more accurate than the RP solution as well as by the observation that this solution becomes singular when the signal power goes to zero. Therefore, it includes a complex RP component $c(z, t)=a(z, t)+j b(z, t)$ and a real LP component $\theta(z, t)$. Equation (5) can also be regarded as a generalization and formalization of the phase-jitter removal concept introduced in [10], with the difference that in (5), the phase jitter is directly determined from the perturbed solution through the time-dependent $\operatorname{term} \theta(z, t)$. The attenuation is implicitly contained in the definition of $u(z, t)$. Substituting (5) in (2), we obtain the complex differential equation

$$
\begin{aligned}
\frac{\partial c}{\partial z} & -j(1+c) \frac{\partial \theta}{\partial z} \\
= & j \frac{\beta_{2}}{2}\left[\frac{\partial^{2} c}{\partial t^{2}}-j(1+c) \frac{\partial^{2} \theta}{\partial t^{2}}-2 j \frac{\partial c}{\partial t} \frac{\partial \theta}{\partial t}-(1+c)\left(\frac{\partial \theta}{\partial t}\right)^{2}\right] \\
& -j \gamma P_{0} \exp (-\alpha z)|1+c|^{2}(1+c),
\end{aligned}
$$

where we have omitted writing the dependence on $z$ and $t$. With respect to the standard RP expansion in (3), the perturbed solution in (5) has one additional degree of freedom (DOF). As a consequence, in (6), we have two equations for three unknown quantities $a, b$, and $\theta$, which makes the system underdetermined. Hence, we have the freedom to arbitrarily add an additional equation to relate these three variables. In principle, all of the choices are equivalent. The difference is that a particular choice can lead to a more useful solution of (6). A trivial choice is to let $\theta(z, t)=\phi_{\mathrm{NL}}(z)$, which turns (5) into the RP expansion of (3), where the perturbation is entirely in the additive term. However, our purpose is to find a linearized model for the perturbation that resembles the LP expansion at high powers and resembles the RP expansion at low powers, with a smooth transition between these regimes. We achieve this goal by seeking an equation for $\theta$ that minimizes the impact of the terms that we are going to neglect in the linearization process. Since the term $\partial \theta / \partial z$ on the left side of (6) is multiplied by the factor $j(1+c)$, we may equate it to the fourth and fifth term on the right side and eliminate the common factor, obtaining

$$
\frac{\partial \theta}{\partial z}=\gamma P_{0} \exp (-\alpha z)|1+c|^{2}+\frac{\beta_{2}}{2}\left(\frac{\partial \theta}{\partial t}\right)^{2} .
$$

By removing the factor $(1+c)$, we have removed the singularity that appears in the CRLP expansion. In this equation, we do not include the term $j(1+c) \partial^{2} \theta / \partial t^{2}$, which does not comply with the constraint that $\theta$ is real. Equation (7) implies that the Kerr term of the NLSE, which corresponds to the last term in (6), directly affects the phase component $\theta$ of the CRLP, which was the intuitive foundation of our approach. The Kerr terms in (6) that are quadratic in $c$ and are neglected in the RP approach become linear in (7) and are included in the CRLP approach, while the cubic term becomes quadratic. The other two equations of the system are obtained by dividing the remaining terms of (6) into real and imaginary parts. Writing explicitly that $c=a+j b$, the system of three real equations for the CRLP terms is

$$
\begin{aligned}
& \frac{\partial a}{\partial z}=\frac{\beta_{2}}{2}\left(-\frac{\partial^{2} b}{\partial t^{2}}+\frac{\partial^{2} \theta}{\partial t^{2}}+a \frac{\partial^{2} \theta}{\partial t^{2}}+2 \frac{\partial a}{\partial t} \frac{\partial \theta}{\partial t}\right) \\
& \frac{\partial b}{\partial z}=\frac{\beta_{2}}{2}\left(\frac{\partial^{2} a}{\partial t^{2}}+b \frac{\partial^{2} \theta}{\partial t^{2}}+2 \frac{\partial b}{\partial t} \frac{\partial \theta}{\partial t}\right) \\
& \frac{\partial \theta}{\partial z}=\gamma P_{0} \exp (-\alpha z)\left(1+2 a+a^{2}+b^{2}\right)+\frac{\beta_{2}}{2}\left(\frac{\partial \theta}{\partial t}\right)^{2} .
\end{aligned}
$$

In (8c), the time-independent term $\gamma P_{0} \exp (-\alpha z)$ produces the nonlinear-phase rotation term $\phi_{\mathrm{NL}}$ of the noise-free solution, 
defined in (4). Hence, we may isolate that term by posing $\theta(z, t)=\phi(z, t)+\phi_{\mathrm{NL}}(z)$. Finally, by neglecting the quadratic terms and denoting the Fourier transforms by uppercase letters of the corresponding quantities in lowercase, we obtain the linearized system of equations for the CRLP model

$$
\begin{aligned}
& \frac{\partial A}{\partial z}=\frac{\beta_{2}}{2} \omega^{2}(B-\Phi) \\
& \frac{\partial B}{\partial z}=-\frac{\beta_{2}}{2} \omega^{2} A, \\
& \frac{\partial \Phi}{\partial z}=2 \gamma P_{0} \exp (-\alpha z) A .
\end{aligned}
$$

For the sake of comparison, we also report the linearized system for the RP model

$$
\begin{aligned}
\frac{\partial A^{\prime}}{\partial z} & =\frac{\beta_{2}}{2} \omega^{2} B^{\prime} \\
\frac{\partial B^{\prime}}{\partial z} & =-\frac{\beta_{2}}{2} \omega^{2} A^{\prime}-2 \gamma P_{0} \exp (-\alpha z) A^{\prime}
\end{aligned}
$$

where $A^{\prime}$ and $B^{\prime}$ are the Fourier transform of $a^{\prime}$ and $b^{\prime}$ in (3). The differences are that in the CRLP we have three rather than two equations, the Kerr term $2 \gamma P_{0} \exp (-\alpha z) A$ has been moved from the equation driving the evolution of $B^{\prime}$ to that one driving the evolution of $\Phi$, and $A$ is also affected by $\Phi$ through dispersion. However, if we define a new variable

$$
Y(z, \omega)=B(z, \omega)-\Phi(z, \omega)
$$

and take the difference of $(9 b)$ and $(9 c)$, we obtain

$$
\begin{aligned}
& \frac{\partial A}{\partial z}=\frac{\beta_{2}}{2} \omega^{2} Y \\
& \frac{\partial Y}{\partial z}=-\frac{\beta_{2}}{2} \omega^{2} A-2 \gamma P_{0} \exp (-\alpha z) A
\end{aligned}
$$

which is the same system as in (10) with $A=A^{\prime}$ and $Y=B^{\prime}$. As a consequence, we may solve (9) by a two-step procedure. First, we exploit the RP solution (derived in [8] and [9] for instance) to evaluate the propagation of $A$ and $Y$. Then, we resort to (9) and (11) to identify how the contribution of the fictitious variable $Y$ has to be shared between the real perturbation terms $B$ and $\Phi$. In particular, when $\alpha=0$, and $\gamma$ and $\beta_{2}$ are constant, the equations in (12) have constant coefficients and can be solved analytically. Defining the vector $\mathbf{X}_{2}(z, \omega)=$ $[A(z, \omega), Y(z, \omega)]^{T}$, the solution of (12) can be expressed in a matrix notation as $\mathbf{X}_{2}(z, \omega)=\mathrm{T}_{\mathrm{RP}}(z, \omega) \cdot \mathbf{X}_{2}(0, \omega)$, where $\mathrm{T}_{\mathrm{RP}}$ is the $2 \times 2$ transfer matrix for the RP model

$$
\mathrm{T}_{\mathrm{RP}}(z, \omega)=\left[\begin{array}{cc}
\cos (k z) & \eta \sin (k z) \\
-\left(\frac{1}{\eta}\right) \sin (k z) & \cos (k z)
\end{array}\right]
$$

with

$$
k=\left[\frac{\beta_{2}}{2} \omega^{2}\left(\frac{\beta_{2}}{2} \omega^{2}+2 \gamma P_{0}\right)\right]^{1 / 2}, \eta=\frac{\beta_{2}}{2} \frac{\omega^{2}}{k} .
$$

Integrating (9b) and (9c) and taking their ratio, we obtain

$$
\frac{B(z, \omega)-B(0, \omega)}{\Phi(z, \omega)-\Phi(0, \omega)}=-\frac{\beta_{2} \omega^{2}}{4 \gamma P_{0}}=\frac{\eta^{2}}{\eta^{2}-1}
$$

which, together with (11), constitutes the linear algebraic system for $B$ and $\Phi$. By defining the vector $\mathbf{X}_{3}(z, \omega)=$ $[A(z, \omega), B(z, \omega), \Phi(z, \omega)]^{T}$, the final solution of (9) can be expressed in matrix notation as $\mathbf{X}_{3}(z, \omega)=\mathrm{T}(z, \omega) \cdot \mathbf{X}_{3}(0, \omega)$, where $T$, shown in (16) at the bottom of this page, is the transfer matrix for the CRLP model. We note that for $\beta_{2}<0$ and $|\omega|<2 \sqrt{\gamma P_{o} /\left|\beta_{2}\right|}$, the parameters $k$ and $\eta$ are imaginary. However, (13)-(16) still hold, and the elements of transfer matrices in (13) and (16) are still real. The ratio in (15) discriminates between two different behaviors of the CRLP model. When nonlinearity dominates over dispersion, that ratio is small, and the perturbation appears primarily in the evolution of $\phi$. On the other hand, when dispersion dominates, that ratio is large and the perturbation appears primarily in the evolution of $b$. For intermediate cases, (15) ensures a balance between RP and LP that enhances the model accuracy. We note that the CRLP approach includes all of the relevant quadratic perturbation terms that are neglected in the RP approach. In fact, all of the neglected terms in (8), either correspond to the cubic terms of (6), or are proportional to the first or second derivative of $\phi$ and to $\beta_{2}$. These last terms would be relevant only at high dispersion and frequency. However, (15) ensures that $\phi$ is not significantly affected by the perturbation at high dispersion and frequency. Finally, we note that in place of (7), we could have set $b(z, t)=0$, mapping all of the perturbation on $a$ and $\phi$ and obtaining a sort of CRLP model with only two real variables. In that case, splitting (6) into real and imaginary parts, after linearization, $a$ and $\phi$ would be driven by the same equations in (12), but with $\Phi=Y$. This model would give similar results to the proposed CRLP model in all cases where the quadrature component $b$ is small compared to the signal. However, it would not behave as the RP model in the linear regime-for which the RP model gives an exact solution-and would be less accurate for small-signal amplitude.

A comparison can also be made with the theory developed in [11] for the zero-frequency component of the RP. According to (10a), the linearized RP theory predicts that the zero-frequency component of the inphase perturbation term $a^{\prime}$ is not affected by parametric gain. However, as pointed out in [11], the quadratic terms neglected in the RP approximation also affect $a^{\prime}$ at zero

$$
\mathbf{T}(z, \omega)=\left[\begin{array}{ccc}
\cos (k z) & \eta \sin (k z) & -\eta \sin (k z) \\
-\eta \sin (k z) & 1+\eta^{2}[\cos (k z)-1] & -\eta^{2}[\cos (k z)-1] \\
\left(\frac{1}{\eta}-\eta\right) \sin (k z) & \left(\eta^{2}-1\right)[\cos (k z)-1] & \eta^{2}-\left(\eta^{2}-1\right) \cos (k z)
\end{array}\right]
$$


frequency. By contrast, as shown in (9c), the linearized CRLP model includes parametric gain at zero frequency through the term $\phi$. According to (5), the phase $\phi$ is responsible for a rotation of the signal in the complex plane. If we project this rotation onto the inphase and quadrature components with respect to the output signal, we obtain a quadrature component that is linear in $\phi$, and an inhase component that is quadratic in $\phi$, in agreement with the behavior predicted in [11].

Finally, compared to the covariance matrix method proposed in [10], the CRLP method formalizes and generalizes the concept of phase-jitter removal. In fact, the phase-jitter removal that is required in the covariance matrix method in order to obtain a Gaussian distribution of the noise and accurate results is contained in (5). In the place of random phase jitter associated with each pulse, which must be extracted during the propagation, we have a random process $\phi(z, t)$, whose propagation is described by the CRLP equations. On the other hand, with respect to [10], the CRLP equations in this paper are only derived for a continuous-wave $\mathrm{CW}$ signal. This limitation is not severe for NRZ systems. However, an extension of the CRLP model to modulated signals could be of interest for narrow RZ signals in which timing jitter becomes the dominant source of signal degradation. This extension is theoretically possible by changing the CRLP ansatz in (5) into

$$
u(z, t)=\sqrt{P_{0}}\left[u_{0}(z, t)+c(z, t)\right] \exp [-j \theta(z, t)]
$$

where $u_{0}(z, t)$ is the normalized noise-free solution of the NLSE.

\section{ASE-Noise Propagation In Multispan Systems}

In this section, we apply the CRLP model to study the propagation of ASE noise in multispan systems. The ASE noise generated by optical amplifiers is the source of a random perturbation, and the inverse Fourier transform $\mathbf{x}_{3}$ of the vector $\mathbf{X}_{3}$ defined in Section II is a vector process, statistically characterized by the $3 \times 3$ power spectral density (PSD) matrix

$$
\mathrm{G}(z, \omega) \triangleq \mathcal{F}_{\tau}\left\{E\left\{\mathbf{x}_{3}(z, t) \mathbf{x}_{3}^{T}(z, t+\tau)\right\}\right\}
$$

where $E\{\cdot\}$ indicates expectation, and $\mathcal{F}_{\tau}\{\cdot\}$ indicates the Fourier transform with respect to $\tau$. Since each amplifier is an independent source of complex additive white Gaussian noise (AWGN), its effect can be modeled by adding two forcing terms to the system of differential equations in (9), which becomes

$$
\begin{aligned}
& \frac{\partial A}{\partial z}=\frac{\beta_{2}}{2} \omega^{2}(B-\Phi)+W_{p} \\
& \frac{\partial B}{\partial z}=-\frac{\beta_{2}}{2} \omega^{2} A+W_{q} \\
& \frac{\partial \Phi}{\partial z}=2 \gamma P_{0} \exp (-\alpha z) A
\end{aligned}
$$

where $W_{p}(z, \omega)$ and $W_{q}(z, \omega)$ are the Fourier transform of the inphase and quadrature component of the complex forcing term $w(z, t) \triangleq \sum_{i} n_{i}(t) \delta\left(z-z_{i}\right)$, where $z_{i}$ is the location of the $i$ th amplifier, and $\delta\left(z-z_{i}\right)$ is the Dirac delta function. In the definition of the forcing term, the $n_{i}(t)$ are independent identically distibuted complex Gaussian random processes, with

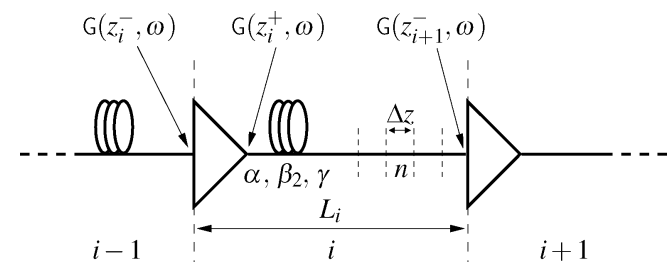

Fig. 1. Generic $i$ th span of a multispan system with optical amplifiers. The span of length $L_{i}$, variable attenuation coefficient $\alpha$, dispersion coefficient $\beta_{2}$, and nonlinear coefficient $\gamma$ are divided into $N$ steps of length $\Delta z=L_{i} / N$ so that in each step, the fiber parameters can be considered constant and the attenuation negligible.

$E\left\{n_{i}(t)\right\}=0$ and $E\left\{n_{i}(t) n_{j}^{*}(t+\tau)\right\}=N_{0} \delta(\tau) \delta_{i j}$, where $N_{0}$ is the PSD of the ASE noise generated by each amplifier on any polarization, and $\delta_{i j}$ is the Kronecker delta function.

Thus, considering the generic $i$ th span of length $L_{i}$ represented in Fig. 1, the PSD matrix at the output of the amplifier is

$$
\mathrm{G}\left(z_{i}^{+}, \omega\right)=\mathrm{G}\left(z_{i}^{-}, \omega\right)+\mathrm{G}_{0}
$$

where $G_{0}=\operatorname{diag}\left\{N_{0} / 2, N_{0} / 2,0\right\}$. In general, the attenuation is not negligible, and the fiber parameters are not constant. Thus, the analytical formula in (16) cannot be directly applied to the whole span. A closed-form solution for the RP model when $\alpha \neq 0$ is given in [9] in terms of Hankel's functions. However, we prefer to follow the simple approach suggested in [8] for the RP model. We divide the span of length $L_{i}$ into $N$ steps of length $\Delta z=L_{i} / N$, as depicted in Fig. 1, such that each step has a negligible attenuation $\alpha \Delta z \simeq 0$, constant dispersion, and an effective nonlinear coefficient $\gamma_{n}=$ $\gamma \exp (-\alpha n \Delta z)[1-\exp (-\alpha \Delta z)] / \alpha \Delta z$. The transfer matrix $\mathrm{T}_{n}(\Delta z, \omega)$ of each step is given by (16), and the overall transfer matrix can be evaluated by multiplying the transfer matrices of each step

$$
\mathrm{T}\left(L_{i}, \omega\right)=\prod_{n=N}^{1} \mathrm{~T}_{n}(\Delta z, \omega) .
$$

The PSD matrix at the output of the span is

$$
\mathrm{G}\left(z_{i+1}^{-}, \omega\right)=\mathrm{T}\left(L_{i}, \omega\right) \mathrm{G}\left(z_{i}^{+}, \omega\right) \mathrm{T}^{T}\left(L_{i}, \omega\right) .
$$

By repeating the procedure described in (20)-(22) for each span composing the system, we finally obtain the output power spectral density (PSD) matrix.

Due to the linearization of our propagation equations, which we carried out by neglecting the quadratic terms in (8), the quantities $a, b$, and $\phi$ at each point in time will remain Gaussian distributed at every $z$ in the presence of AWGN. However, the signal itself, given by a nonlinear combination of $a, b$, and $\phi$ in (5), will not be Gaussian distributed in general. Thus, the output process is not Gaussian but it turns out to be a combination of Gaussian processes. With respect to RP, the CRLP approach is slightly more complex, because the power spectral density is described by a $3 \times 3$ rather than by a $2 \times 2$ matrix. However, we will show that the accuracy of the CRLP is significantly higher than the RP, since it accounts for the effects of the Kerr interactions on $\phi$ that are neglected in the RP. 


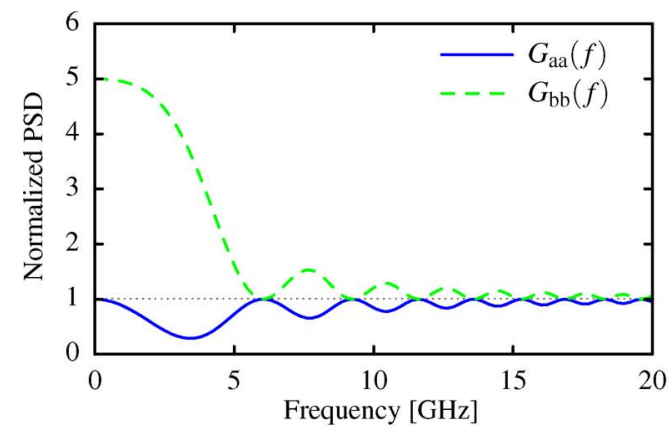

(a)

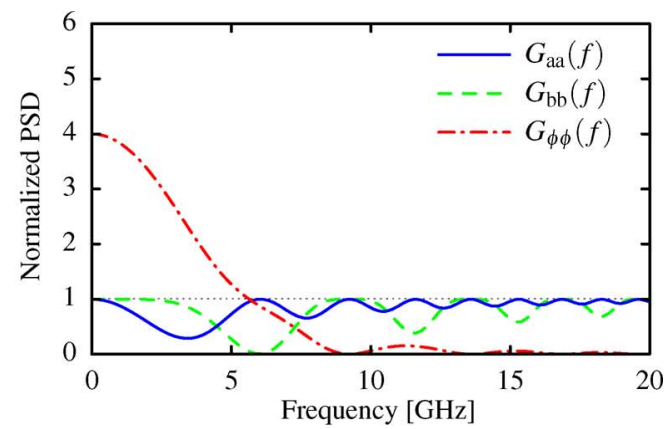

(b)

Fig. 2. Normalized PSD matrix (diagonal elements) of the (a) RP model and (b) CRLP model for $P_{0}=10 \mathrm{~mW}, D=-50 \mathrm{ps} /(\mathrm{nm} \cdot \mathrm{km}), \gamma=2(\mathrm{~W} \cdot \mathrm{km}){ }^{-1}$, and $L=50 \mathrm{~km}$, corresponding to normal dispersion and $\phi_{\mathrm{NL}}=1 \mathrm{rad}$.

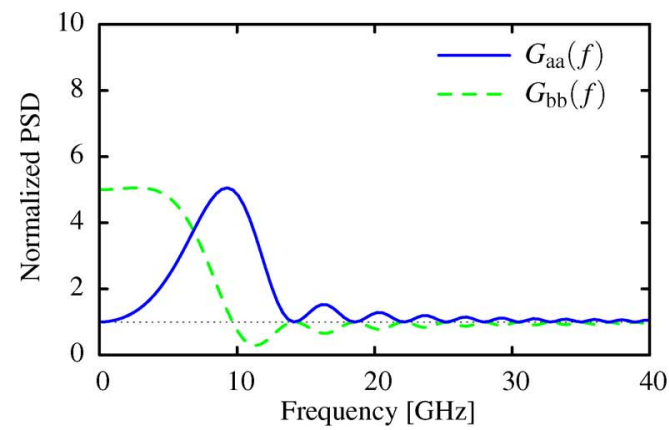

(a)

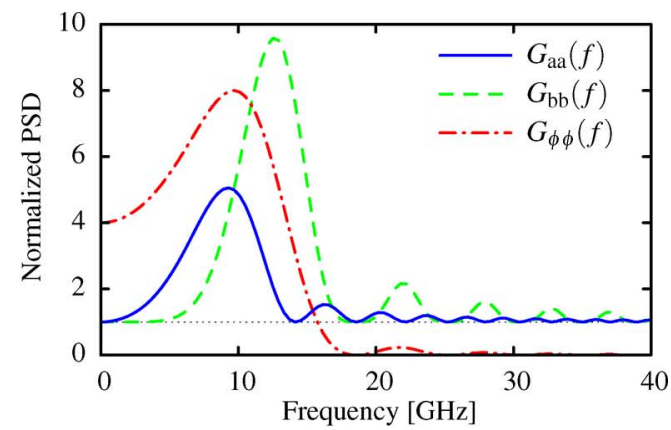

(b)

Fig. 3. Normalized PSD matrix (diagonal elements) of the (a) RP model and (b) CRLP model for $P_{0}=10 \mathrm{~mW}, D=17 \mathrm{ps} /(\mathrm{nm} \cdot \mathrm{km}), \gamma=2(\mathrm{~W} \cdot \mathrm{km})^{-1}$, and $L=50 \mathrm{~km}$, corresponding to anomalous dispersion and $\phi_{\mathrm{NL}}=1 \mathrm{rad}$.

\section{APPLICATIONS OF THE CRLP MOdEL to Simple Systems}

Fig. 2 shows a comparison between the main (diagonal) terms of the output PSD matrices for the (a) RP and (b) CRLP models in the case of a CW plus additive white Gaussian noise (AWGN) propagating through a lossless fiber with $P_{0}=10 \mathrm{~mW}, D=$ $-50 \mathrm{ps} /(\mathrm{nm} \cdot \mathrm{km}), \gamma=2(\mathrm{~W} \cdot \mathrm{km})^{-1}, L=50 \mathrm{~km}$. These choices correspond to normal dispersion and $\phi_{\mathrm{NL}}=1 \mathrm{rad}$. The dispersion parameter $D=-2 \pi c \beta_{2} / \lambda^{2}$, where $c$ is the speed of light and $\lambda$ is the reference wavelength, is often used to characterize the fiber dispersion. The PSDs are normalized with respect to the PSD of the inphase or quadrature component of the injected AWGN noise. Comparing (10) and (12), we infer that the PSD of the inphase component $G_{a a}$ is the same for the RP and CRLP model, while the PSD of the quadrature component $G_{b b}$ is different. Moreover, the phase-noise PSD of the CRLP model in Fig. 2(b), $G_{\phi \phi}$, is concentrated at low frequencies. This result is the consequence of (15), which implies that $\phi$ is primarily affected by parametric gain at low frequencies, while $b$ is primarily affected by parametric gain at higher frequencies. Fig. 3 shows the same comparison as in Fig. 2, but for an anomalous dispersion $D=17 \mathrm{ps} /(\mathrm{nm} \cdot \mathrm{km})$. Those results can be generalized by showing that according to the CRLP model, the sign of the dispersion determines how $G_{a a}$ and $G_{b b}$ are affected by parametric gain. In an uncompensated span, $a$ and $b$ are both subject to attenuation or amplification when the dispersion is normal $D<0$, or anomalous $D>0$, respectively, and are both unaffected at the carrier frequency or when $D=0$. On the other hand, the RP model predicts a different behavior for $b$, whose large increase at the carrier frequency is often responsible for an increase in the marks at the detection point in the receiver and, thus, for BER improvement.

Another interesting comparison can be drawn in the case where there is no dispersion, for which an exact solution exists. Letting $\beta_{2}=0$ in (8), (9), or (10), or letting also $\gamma=0$ in (10), we obtain, respectively

$$
\begin{aligned}
& \text { Exact: }\left\{\begin{array}{l}
a(z)=a(0) \\
b(z)=b(0) \\
\phi(z)=\phi(0)+\left[2 a(0)+a^{2}(0)+b^{2}(0)\right] \phi_{\mathrm{NL}}
\end{array}\right. \\
& \text { CRLP }:\left\{\begin{array}{l}
a(z)=a(0) \\
b(z)=b(0) \\
\phi(z)=\phi(0)+2 a(0) \phi_{\mathrm{NL}}
\end{array}\right. \\
& \mathrm{RP}:\left\{\begin{array}{l}
a(z)=a(0) \\
b(z)=b(0)-2 a(0) \phi_{\mathrm{NL}}
\end{array}\right. \\
& \text { AWGN : }\left\{\begin{array}{l}
a(z)=a(0) \\
b(z)=b(0)
\end{array}\right.
\end{aligned}
$$

where we dropped the time dependence because it is irrelevant in this case. When there is no dispersion, the linearized CRLP model yields the exact amplitude and a first-order approximation of the phase, the RP model yields the wrong amplitude and phase, and the AWGN model yields the correct amplitude, but an incorrect phase. For instance, if we consider a system with $\phi_{\mathrm{NL}}=2 \mathrm{rad}$, and initial conditions $a(0)=0.2, b(0)=\phi(0)=$ 0 , we obtain the solutions presented in Fig. 4. As can be seen, the CRLP yelds the most accurate result. Moreover, if we are 


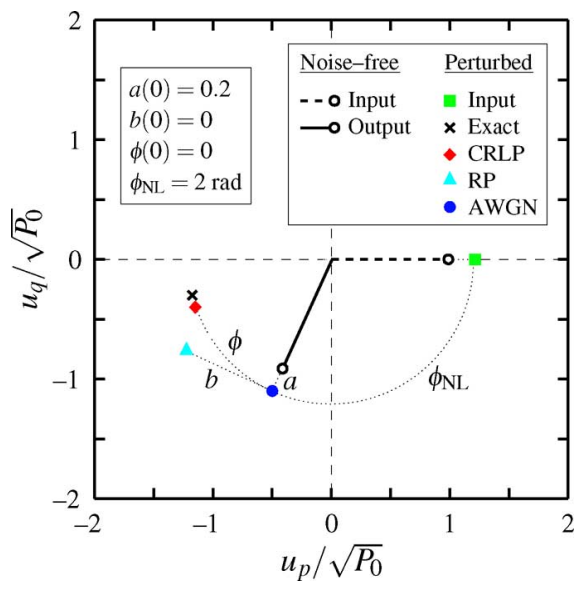

Fig. 4. Schematic representation of the zero-dispersion solutions in (23). Thick lines represent the input and output noise-free signals at a generic time $t$. Symbols represent the corresponding noisy input and output (exact, AWGN, RP, and CRLP) perturbed signals. Thin dotted lines show how the perturbed signals relate to the noise-free signals through the perturbation components.

mainly interested in the output signal amplitude, as in the case of OOK systems, the AWGN approximation is better than the $\mathrm{RP}$, even though the phase is incorrect.

When there is no dispersion, the CRLP solution in (23b) reproduces the well-known results for the phase noise in amplified systems [13]-[15]. Considering the inphase and quadrature components of the injected ASE noise, $a(0, t)$ and $b(0, t)$, respectively, as the source of the perturbation, the phase $\phi(z, t)$ corresponds to the nonlinear phase noise, which, at first order, is proportional to $a(0, t)$ and is therefore Gaussian distributed, although its exact distribution slightly deviates from Gaussian if we include the quadratic components. This result confirms the accuracy of the CRLP model, which exactly predicts the well-known results for the nonlinear phase noise at zero dispersion and only leads to a slight discrepancy with the signal phase. Indeed, the model is valid even in the presence of dispersion, in which case it predicts a parametric gain effect for $a$ and $b$ and a limited bandwidth in which phase noise is generated, as shown in Fig. 2. Finally, we note that in the linear regime, the RP and CRLP models converge to the AWGN model and give exact results, as can be seen by letting $\gamma=0$ (or, equivalently, $\left|\beta_{2}\right| \rightarrow \infty$ ) in (13) and (16), respectively. In this sense, we can view the CRLP model as a combination of the theories developed for the parametric gain effect [8]-[11] and the nonlinear phase noise [13]-[15].

\section{APPLiCATIONS OF THE CRLP MODEL}

In this section, we give some applications of the CRLP model. Under the aforementioned CW approximation, the PSD matrix of the CRLP model in (18) gives a complete statistical characterization of the optical signal at the receiver for a multispan optically amplified single-channel system. Therefore, the proposed model is suitable for the analysis of single-channel optical systems regardless of the detection strategy and modulation format (provided that the $\mathrm{CW}$ approximation holds). In regards to the detection strategy, we apply the CRLP model to the evaluation of the pdf of the optical sample for a coherent receiver, and to the evaluation of the pdf of the photodetected sample for a direct-detection receiver. In regards to modulation formats, we apply the model to the evaluation of the BER for an NRZ-OOK system. In order to check the validity of the model and the improvement in accuracy with respect to other models, all of the results are compared to the simple AWGN model, the classical RP model, and numerical simulations, which are performed by using the multicanonical Monte Carlo (MMC) algorithm described in [16]-[18]. Finally, we shortly discuss some possible extensions to different modulation formats and wavelength-division multiplexing (WDM) systems.

\section{A. Probability Density Function of the Optical Signal}

We consider the propagation of a CW signal plus AWGN noise in a lossless fiber and look at the pdf of the optical signal after propagation. This example is of particular interest for the analysis of a coherent receiver, where the detected samples are proportional to the optical field. In addition, it allows us to understand the behavior of the CRLP model and its ability to obtain the non-Gaussian distribution of the optical noise after propagation. Fig. 5 shows the contour plot of the joint pdf $p_{u_{p}, u_{q}}\left(u_{p}, u_{q}\right)$, where $u_{p}$ and $u_{q}$ are the inphase and quadrature components of the optical signal $u(z, t)=u_{p}(z, t)+j u_{q}(z, t)$ at the output of the fiber. The pdf is independent of time since the process is stationary. In the AWGN or RP model, $u(z, t)$ is given by (3). In the AWGN case, the interaction between noise and signal is completely neglected, $u_{p}$ and $u_{q}$ are independent identically distributed Gaussian random variables, and the contour lines of the pdf have a circular shape. In the RP case, $u_{p}$ and $u_{q}$ are still Gaussian because of linearization, but are not independent because of parametric gain. Their covariance matrix can be derived from the PSD matrix, and the contour lines assume a slightly tilted, elliptical shape. In the CRLP model, $u(z, t)$ is given by (5). Due to the nonlinear dependence of $u$ on $\phi$, the contour lines bend around the origin, giving a banana-like shape. Consequently, the Cartesian fields $u_{p}$ and $u_{q}$ are no longer Gaussian. In this case, the joint pdf can be evaluated as explained in Appendix A. Finally, simulations are made by using the split-step Fourier method for the propagation of the CW plus AWGN, and applying the MMC algorithm described in [16] and [17] to estimate the pdf with uniform accuracy down to low values.

Fig. 5 shows two different cases. in Fig. 5(a), the CW power is $P_{0}=20 \mathrm{~mW}$, the noise PSD is $N_{0}=1.6 \cdot 10^{-14} \mathrm{~W} / \mathrm{Hz}$, corresponding to an optical signal-to-noise ratio ${ }^{1} \mathrm{OSNR}_{0.1 \mathrm{~nm}} \simeq$ $14 \mathrm{~dB}$ for an ideal $10 \mathrm{~Gb} / \mathrm{s}-\mathrm{NRZ}$ signal with the same peak power, and the fiber dispersion is $D=-5 \mathrm{ps} /(\mathrm{nm} \cdot \mathrm{km})$; in Fig. 5(b), $P_{0}=5 \mathrm{~mW}, N_{0}=4 \cdot 10^{-} 15 \mathrm{~W} / \mathrm{Hz}$, corresponding to the same $\operatorname{OSNR}_{0.1 \mathrm{~nm}} \simeq 14 \mathrm{~dB}$, and $D=5 \mathrm{ps} /(\mathrm{nm} \cdot \mathrm{km})$. In both cases, the nonlinear coefficient is $\gamma=2(\mathrm{~W} \cdot \mathrm{km})^{-1}$, the fiber length is $L=50 \mathrm{~km}$, and the noise bandwidth is limited by a Gaussian filter with a 20-GHz 3-dB bandwidth. In the graphs, the fields $u_{p}$ and $u_{q}$ are normalized with respect to $\sqrt{P_{0}}$. The large deviation of the MMC simulations from the AWGN model shows that signal-noise interaction is strong. In particular, in the normal dispersion regime of Fig. 5(a), with a fairly high value $\phi_{\mathrm{NL}}=2 \mathrm{rad}$ of the nonlinear phase rotation, two

\footnotetext{
${ }^{1}$ The reported OSNR values also take the noise in the orthogonal polarization into account.
} 


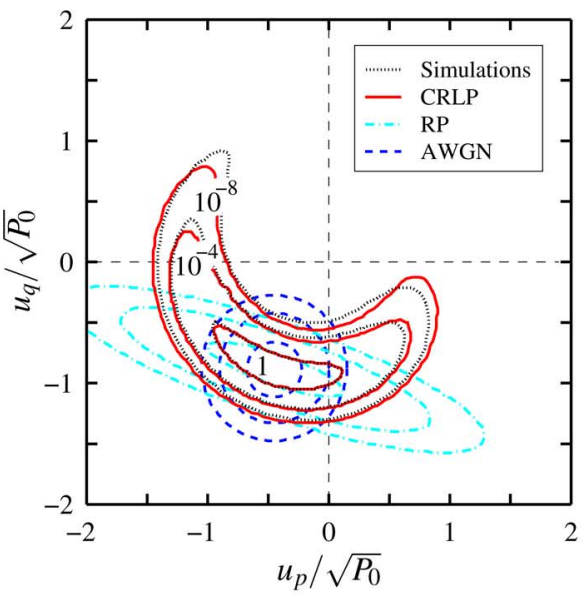

(a)

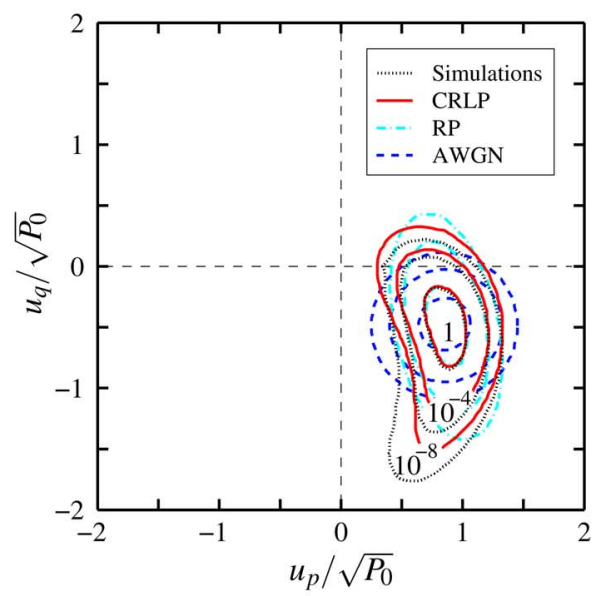

(b)

Fig. 5. Pdf of the optical signal after propagation through a single lossless span according to the AWGN, RP, and CRLP models, and to MMC simulations: (a) $P_{0}=20 \mathrm{~mW}, D=-5 \mathrm{ps} /(\mathrm{nm} \cdot \mathrm{km}), \gamma=2(\mathrm{~W} \cdot \mathrm{km})^{-1}, L=50 \mathrm{~km}, N_{0}=1.6 \cdot 10^{-14} \mathrm{~W} / \mathrm{Hz}$, corresponding to $\phi_{\mathrm{NL}}=2 \mathrm{rad}$ and OSNR $0.1 \mathrm{~nm} \simeq 14 \mathrm{~dB}$; (b) $P_{0}=5 \mathrm{~mW}, D=5 \mathrm{ps} /(\mathrm{nm} \cdot \mathrm{km}), \gamma=2(\mathrm{~W} \cdot \mathrm{km})^{-1}, L=50 \mathrm{~km}, N_{0}=4 \cdot 10^{-15} \mathrm{~W} / \mathrm{Hz}$, corresponding to $\phi_{\mathrm{NL}}=0.5 \mathrm{rad}$ and OSNR $0.1 \mathrm{~nm} \simeq 14 \mathrm{~dB}$.

effects are evident: 1) shrinkage of the noise pdf along the signal direction, and 2) strong bending of the pdf around the origin. The first effect, which corresponds to a reduction of the inphase noise component $a$, is correctly predicted by the RP and CRLP model, as shown in Fig. 2, while the second effect, which is related to the presence of strong phase noise, is correctly predicted only by the CRLP model. The agreement of the CRLP model with simulations is excellent for small perturbations, as evidenced by the inner contour line, and is good even for large perturbations, as evidenced by the outer contour lines. The quadratic terms $a^{2}$ and $b^{2}$, which are neglected in the linearization of the third equation of (8), produce a slight asymmetry of $\phi$ and a slight increase of its variance, which explains the discrepancy between MMC and CRLP on the tips of the outer contour lines. This phase discrepancy is only observed for perturbations that are larger than the signal itself, and are not expected to be relevant in amplitude-modulated systems. On the other hand, the RP model greatly overestimates the quadrature component $b$, as shown in Fig. 2, and it does not predict the conversion of amplitude noise into phase noise. Therefore, we expect the RP model to overestimate the performance of both amplitude- and phase-modulated systems. In the anomalous dispersion regime of Fig. 2(b) , the CRLP model again shows the best agreement with simulations of all the perturbation methods that we studied, but the agreement is not as good as in Fig. 2(a) . In fact, in this case, the parametric gain effect, contrary to what happens in case 1), tends to transfer the power from signal to noise, as shown in Fig. 3, with a fast increase of the perturbation and a consequent degradation of the model accuracy. Already at the value $\phi_{\mathrm{NL}}=0.5 \mathrm{rad}$ of Fig. 3(b), the discrepancy for large perturbations is significant. However, as we will show in the following examples, the discrepancy is only due to a phase error, and the amplitude, which is relevant for amplitude-modulated systems, is still correctly predicted.

\section{B. Probability Density Function of the Photodetected Signal}

As in the previous example, we consider again the simple case of CW plus AWGN noise propagating through a lossless fiber, but now we focus attention on the pdf of the signal after a standard OOK receiver. After propagation, the optical signal is filtered by a bandpass Gaussian filter with 3-dB bandwidth of $20 \mathrm{GHz}$, photodetected and filtered again by a low-pass fifth-order Bessel filter with a 3-dB bandwidth of $7.5 \mathrm{GHz}$. Fig. 6(a)-(c) shows the pdfs of the normalized photocurrent for three different cases: Fig. 6(a) shows a normal dispersion fiber $D=-50 \mathrm{ps} /(\mathrm{nm} \cdot \mathrm{km})$ with $\mathrm{CW}$ power $P_{0}=20 \mathrm{~mW}$ and a noise PSD $N_{0}=1.6 \cdot 10^{-14} \mathrm{~W} / \mathrm{Hz} ;$ Fig. 6(b) shows an anomalous dispersion fiber $D=17 \mathrm{ps} /(\mathrm{nm} \cdot \mathrm{km})$ with $P_{0}=$ $10 \mathrm{~mW}$ and $N_{0}=8 \cdot 10^{-15} \mathrm{~W} / \mathrm{Hz}$; and Fig. 6(c) shows a zero dispersion fiber with $P_{0}=5 \mathrm{~mW}$ and $N_{0}=4 \cdot 10^{-15} \mathrm{~W} / \mathrm{Hz}$. In all three cases, the fiber length is $L=50 \mathrm{~km}$, the nonlinear coefficient is $\gamma=2(\mathrm{~W} \cdot \mathrm{km})^{-1}$, and $\mathrm{OSNR}_{0.1 \mathrm{~nm}} \simeq 14 \mathrm{~dB}$ as in the previous examples. The pdfs are derived from the PSD matrices for the AWGN, RP, or CRLP models, as explained in Appendix B, and are then compared to MMC simulations. In all cases, the CRLP model is in good agreement with simulations, which strongly deviate from the AWGN prediction due to the nonlinear interaction between the noise and signal. In particular, the left tail of the pdf, which is the most important for the evaluation of the BER, in Fig. 6(a)) and Fig. 6(b) differs substantially from the simulations in the AWGN case, mainly due to a change of the inphase component $a$ of the perturbation, that is predicted by the RP and CRLP models, as shown in Figs. 2 and 3. However, the RP model also predicts a strong increase of the quadrature component $b$, leading to a systematic biasing of the pdf toward large values of the photocurrent. This bias is evident in the left and right tails of the pdfs. In particular, in Fig. 6(b), the increase of the inphase component, which leads to a small increase of the left tail of the pdf in its upper portion, is masked by the larger increase of the quadrature component, which leads to a significant decrease of the same tail at lower values. As a result, the RP model would predict an improvement of performance in the anomalous dispersion regime, where the CRLP model correctly predicts the strong degradation shown by the MMC simulations.

In Fig. 6(c), a completely different behavior is observed. As already pointed out in Section IV, at zero dispersion, only the phase term $\phi$ of the CRLP model is affected by PG, leaving the 


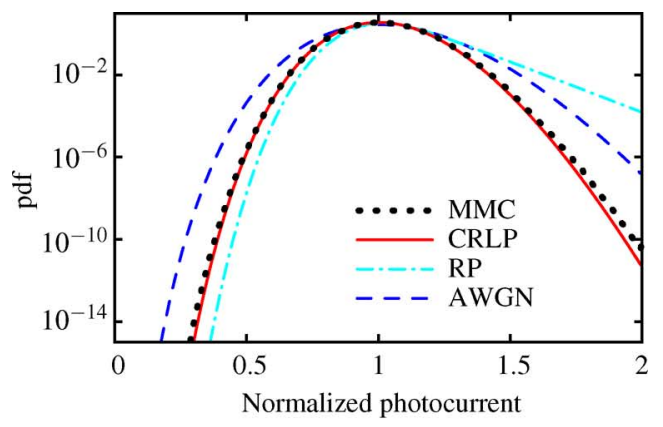

(a)

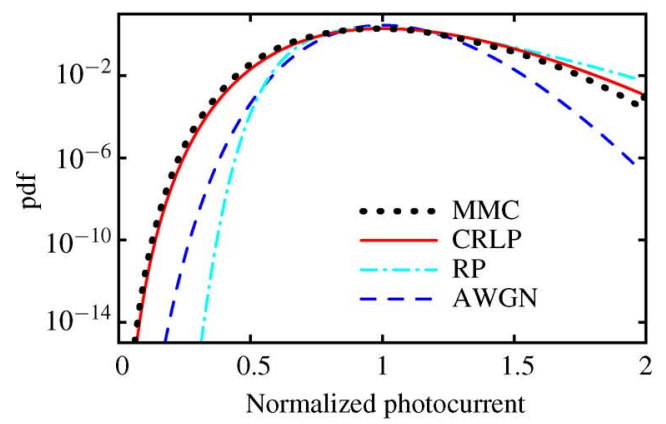

(b)

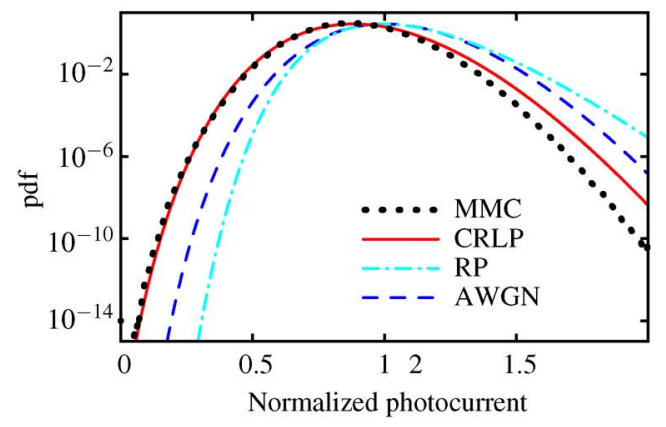

(c)

Fig. 6. Pdf of the normalized photocurrent after propagation through a single lossless span with $L=50 \mathrm{~km}$ and $\gamma=2(\mathrm{~W} \cdot \mathrm{km})^{-1}$, according to the AWGN, RP, and CRLP models, and to MMC simulations: (a) $P_{0}=20 \mathrm{~mW}$, $D=-50 \mathrm{ps} /(\mathrm{nm} \cdot \mathrm{km}), N_{0}=1.6 \cdot 10^{-14} \mathrm{~W} / \mathrm{Hz}$, corresponding to $\phi_{\mathrm{NL}}=$ 2 and $\mathrm{OSNR}_{0.1 \mathrm{~nm}} \simeq 14 \mathrm{~dB}$; (b) $P_{0}=10 \mathrm{~mW}, D=17 \mathrm{ps} /(\mathrm{nm} \cdot \mathrm{km})$, $N_{0}=8 \cdot 10^{-15} \mathrm{~W} / \mathrm{Hz}$, corresponding to $\phi_{\mathrm{NL}}=1$ and $\mathrm{OSNR}_{0.1} \mathrm{~nm} \simeq 14 \mathrm{~dB}$; (c) $P_{0}=5 \mathrm{~mW}, D=0 \mathrm{ps} /(\mathrm{nm} \cdot \mathrm{km}), N_{0}=4 \cdot 10^{-15} \mathrm{~W} / \mathrm{Hz}$, corresponding to $\phi_{\mathrm{NL}}=0.5$ and $\mathrm{OSNR}_{0.1} \mathrm{~nm} \simeq 14 \mathrm{~dB}$.

amplitude unaltered. This case was already investigated in [14], where it is shown that at zero dispersion, the Kerr nonlinearity of the fiber produces an increase in the noise background which, however, does not affect the amplitude noise of the propagating field and, consequently, the performance of an OOK system, unless optical filtering is performed. On the other hand, when optical filtering is performed, significant signal attenuation is observed that depends on the total bandwidth of the ASE noise before filtering. For instance, in the case of Fig. 6(c), the total bandwidth of the injected ASE noise was $320 \mathrm{GHz}$, compared to the $20-\mathrm{GHz}$ bandwidth of the optical filter, which gives significant attenuation. This phenomenon is not captured by either the AWGN or the RP model, but can be explained by the CRLP model. In fact, at zero dispersion, the PG bandwidth is theoretically unlimited in the cases treated here. Therefore, the op- tical filter bandwidth is much narrower than the bandwidth of the phase term $\phi$, and the linear filtering approximation discussed in Appendix B is no longer valid. While a CW signal would pass unaltered through the optical filter, the phase modulation imposed by $\phi$ in the zero-dispersion regime increases the bandwidth of the $\mathrm{CW}$ signal, which is then attenuated by the optical filter. Following [19] and considering a second-order approximation, it can be shown that the normalized intensity $I(t)$ of the phase-modulated CW signal after an optical filter with an impulse response $h(t)$ can be approximated as

$$
I(t) \simeq 1+\log [I(t)] \simeq 1+[\phi(t) \otimes h(t)]^{2}-\phi^{2}(t) \otimes h(t)
$$

where $\otimes$ indicates convolution. Taking the expected value of (24) and exploiting the linearity of expected values to arbitrary linear operators, we obtain

$$
E\{I(t)\} \simeq 1+E\left\{[\phi(t) \otimes h(t)]^{2}\right\}-E\left\{\phi^{2}(t)\right\} \otimes h(t) .
$$

Since $\phi(t)$ is a stationary ergodic process, the expected values on the right side of (25) correspond to the power of $\phi \otimes h$ and the power of $\phi$ (assuming $H(0)=1$ ), respectively, and are independent of time. Therefore, (25) can be rewritten as

$$
E\{I(t)\} \simeq 1-\int_{-\infty}^{\infty} G_{\phi \phi}(\omega)\left[H(0)-|H(\omega)|^{2}\right] d \omega
$$

where $G_{\phi \phi}(\omega)$ is the PSD of the $\phi$ term (i.e., the (3,3) element of the PSD matrix G defined in (18), and $H(\omega)$ is the Fourier transform of $h(t)$ ). When including the signal attenuation given by the integral in (26), which corresponds to a translation of the pdf toward low values, the CRLP model in Fig. 6(c) yields a good approximation of the pdf once more. We note that (25) can be used also for nonzero dispersion. However, in most practical cases, the bandwidth of $G_{\phi \phi}$ is narrower than the optical filter due to dispersion, and the attenuation given by the integral in (25) is negligible.

\section{BER of NRZ-OOK Systems}

Here, we evaluate the bit-error ratio of a simple $10-\mathrm{Gb} / \mathrm{s}$ NRZ-OOK system model with five spans of fiber. Each span has an optical amplifier, $128 \mathrm{~km}$ of standard transmission fiber, and $16 \mathrm{~km}$ of dispersion compensating fiber. The amplifier has a spontaneous emission factor $\eta_{s p}=4$ and the gain is equal to the loss of the entire span. The standard transmission fiber has a dispersion $D=16 \mathrm{ps} /(\mathrm{nm} \cdot \mathrm{km})$, a nonlinear coefficient $\gamma=2(\mathrm{~W} \cdot \mathrm{km})^{-1}$, and attenuation $\alpha=0.22 \mathrm{~dB} / \mathrm{km}$. The dispersion compensating fiber has a dispersion $D=-128 \mathrm{ps} /(\mathrm{nm} \cdot \mathrm{km})$ and the same $\gamma$ and $\alpha$ as the standard transmission fiber. Since we are not interested in the dispersion map optimization, the dispersion in each span is fully compensated. The receiver model has a Gaussian bandpass optical filter with 50-GHz bandwidth, a photodetector, and a fifth-order low-pass Bessel filter with 7.5-GHz bandwidth. The BER is evaluated as explained in Appendix B, and thermal and shot noise are neglected. Simulations are performed by using a version of the MMC algorithm that accounts for pattern dependences [18]. Fig. 7(a) shows the BER as a function of the peak power (measured at the output of each amplifier) 


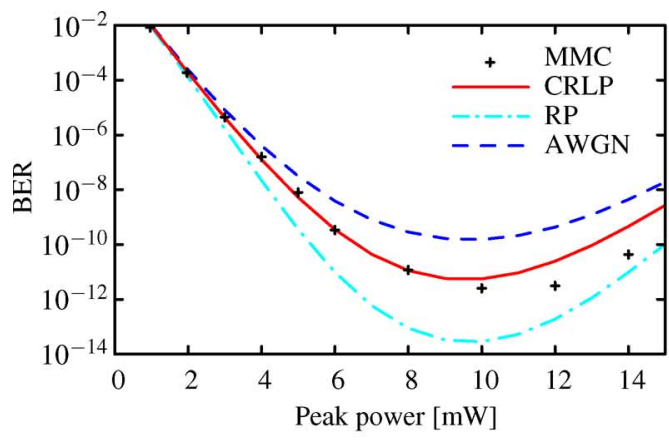

(a)

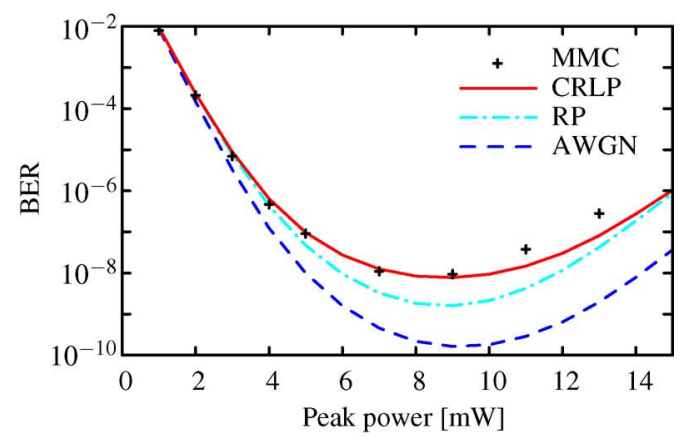

(b)

Fig. 7. BER versus peak power for a five-span 10-Gb/s-NRZ system. (a) $\eta_{s p}=4, \gamma=2(\mathrm{~W} \cdot \mathrm{km})^{-1}, \alpha=0.22 \mathrm{~dB} / \mathrm{km}, 128 \mathrm{~km}$ of transmission fiber with $D=+16 \mathrm{ps} /(\mathrm{nm} \cdot \mathrm{km})$ and $16 \mathrm{~km}$ of compensation fiber with $D=-128 \mathrm{ps} /(\mathrm{nm} \cdot \mathrm{km})$. (b) Transmission and compensation fibers are swapped.

by using the AWGN, RP, CRLP, and MMC algorithms. As explained in Appendix B, self-phase modulation, which is the most relevant nonlinear effect in this case, is taken into account in all of the models by propagating the noise-free signal with the split-step Fourier method. When increasing the peak power, the OSNR increases, but self-phase modulation becomes stronger. As a consequence, the BER curves have a minimum at the point where the best tradeoff between OSNR and self-phase modulation impairments occurs. In addition, the system performance is affected by parametric gain due to the nonlinear interaction between the noise and signal, which is neglected by the AWGN model and approximated differently by the RP and CRLP models. In this example, parametric gain is first visible at $\sim 2 \mathrm{~mW}$, where the various models and the simulations begin to disagree. The BER improvement shown by simulations with respect to the AWGN model is correctly predicted by the CRLP model, which is in agreement with simulations up to $\sim 9 \mathrm{~mW}$ (the minimum of the BER curve), which corresponds to a nonlinear phase rotation $\phi_{\mathrm{NL}} \simeq 1.8 \mathrm{rad}$. By contrast, the BER improvement predicted by the RP model is too large.

Fig. 7(b) shows a different case, where the system characteristics are the same as in (a), but the transmission and compensating fibers are swapped. Although the performance predicted by the AWGN model is almost the same as in the previous case, the actual performance found in the MMC simulations shows completely different behavior due to the parametric gain. In fact, the performance is worse than that predicted by the AWGN model. Again, the agreement between CRLP and simulations is good up to the minimum of the BER curve at $\sim 9 \mathrm{~mW}$, while the RP model is too optimistic.

\section{Extension to Different Modulation Formats and WDM Systems}

The CW approximation adopted in this paper relies on the hypothesis that, during propagation, the noise interacts with a slowly time-varying signal. In the last example, we have shown that this is a reasonable approximation for an NRZ-OOK system. However, it could be too severe if we were interested in the statistical analysis of a rapidly time-varying signal, as in the case of RZ formats or in the case of oversampled receivers. In this case, analogous but more complex equations for the perturbation terms $a, b$, and $\phi$ that also account for the modulation of the interacting signal could be derived by removing the $\mathrm{CW}$ approximation in (5) by using (17) as a starting point.

Another interesting extension of the CRLP model would be the inclusion of other sources of perturbation (noise) that are typical of WDM systems by adding proper forcing terms to (19). For instance, cross-phase modulation (XPM) could be approximately modeled by adding a Gaussian forcing term to (19c), and four-wave mixing (FWM) by adding two more Gaussian forcing terms to (19a) and (19b). The propagation equations would remain as a system of linear differential equations, but-with the forcing terms distributed along $z$-would be nonhomogeneous and require a slightly different procedure for the evaluation of the output PSD matrix. However, all of the perturbation terms would remain Gaussian after propagation, meaning that the PSD matrix in (18) would still give a compete statistical characterization of the optical signal and that the procedures described in this section for pdf or BER evaluation would remain unchanged.

\section{CONCLUSION}

We have introduced a novel perturbation method for the NLSE that reduces to the RP expansion at low power and to the LP expansion at high power. It is more accurate than either. In this CRLP expansion, the optical signal is represented as a perturbed noise-free signal, and the perturbation is described by two additive terms and one phase factor. The phase factor can also be viewed as a generalization and formalization of the phase jitter removal concept introduced in [10].

We have derived the CRLP propagation equations, and we have applied them to amplified multispan optical systems. In this case, the CRLP model can accurately model parametric gain, including the contribution of the Kerr terms that are quadratic with respect to the perturbation, and the nonlinear phase-noise generation. We have compared the results to those predicted by other linearization models, and the CRLP performance was consistently better.

Finally, we have shown how the CRLP model can be applied to evaluate the pdf of the received optical signal in a coherent receiver, the pdf of the postdetection signal in a direct-detection receiver, and the BER of an NRZ-OOK system. We have considered several different examples of pdf or BER evaluation. All of the results obtained with the CRLP model have been compared to other linearization models and to multicanonical Monte Carlo simulations in order to validate the accuracy of the CRLP 
model and to show its advantages. In addition, we have shortly discussed the possibility of removing the $\mathrm{CW}$ approximation and extending the model to the analysis of WDM systems.

\section{APPENDIX A}

In this Appendix, we explain how to derive the pdf of the optical signal $u(z, t)=u_{p}(z, t)+j u_{q}(z, t)$ from the PSD matrix $\mathrm{G}(z, \omega)$ by following the rules for functions of random variables given in [20]. At a fixed time $t$ and position $z$, the perturbation terms $a, b$, and $\phi$ of the CRLP are three Gaussian random variables with multivariate normal distribution

$$
p_{a, b, \phi}(a, b, \phi)=\frac{1}{\sqrt{8 \pi^{3}|\mathrm{~K}|}} \exp \left\{-\frac{1}{2}[a, b, \phi] \mathrm{K}^{-1}[a, b, \phi]^{T}\right\}
$$

whose covariance matrix $\mathrm{K}$ is

$$
\mathrm{K}=\left.\mathcal{F}_{\omega}^{-1}\{\mathrm{G}(z, \omega)\}\right|_{\tau=0} .
$$

From (5), we derive the transformation

$$
\begin{aligned}
u_{p} & =(1+a) \cos \left(\phi+\phi_{\mathrm{NL}}\right)+b \sin \left(\phi+\phi_{\mathrm{NL}}\right) \\
u_{q} & =-(1+a) \sin \left(\phi+\phi_{\mathrm{NL}}\right)+b \cos \left(\phi+\phi_{\mathrm{NL}}\right) \\
\xi & =\phi+\phi_{\mathrm{NL}}
\end{aligned}
$$

where we have introduced the auxiliary variable $\xi$. The corresponding inverse transformation is

$$
\begin{aligned}
a & =u_{p} \cos \xi-u_{q} \sin \xi-1 \\
b & =u_{p} \sin \xi+u_{q} \cos \xi \\
\phi & =\xi-\phi_{\mathrm{NL}} .
\end{aligned}
$$

Since the determinant of the Jacobian matrix of the transformation in (A4) is equal to one, we obtain the joint pdf $p_{u_{p}, u_{q}, \xi}\left(u_{p}, u_{q}, \xi\right)$ by substituting (A4) into (A1). Taking apart the Gaussian factor coming from the $\phi^{2}$ term in brackets in (A1), we write the pdf as

$$
\begin{aligned}
p_{u_{p}, u_{q}, \xi} & \left(u_{p}, u_{q}, \xi\right) \\
= & \frac{1}{\sqrt{2 \pi} \sigma_{\xi}} e^{-\left(\xi-\phi_{\mathrm{NL}}\right)^{2} /\left(2 \sigma_{\xi}^{2}\right)} p_{u_{p}, u_{q}, \xi}^{\prime}\left(u_{p}, u_{q}, \xi\right)
\end{aligned}
$$

where $p_{u_{p}, u_{q}, \xi}^{\prime}\left(u_{p}, u_{q}, \xi\right)$ is the remaining factor, and $\sigma_{\xi}=$ $\left(\mathrm{K}_{3,3}^{-1}\right)^{-1 / 2}$. We finally obtain the desired joint pdf by eliminating the auxiliary variable $\xi$ in (A5) through calculation of the marginal distribution

$$
\begin{aligned}
& p_{u_{p}, u_{q}}\left(u_{p}, u_{q}\right)=\int_{-\infty}^{\infty} p_{u_{p}, u_{q}, \xi}\left(u_{p}, u_{q}, \xi\right) d \xi \\
& \simeq \frac{1}{\sqrt{\pi}} \sum_{k=1}^{N} w_{k} p_{u_{p}, u_{q}, \xi}^{\prime}\left(u_{p}, u_{q}, \sqrt{2} \sigma_{\xi} x_{k}+\phi_{\mathrm{NL}}\right)
\end{aligned}
$$

where $w_{k}$ and $x_{k}, k=1, \ldots, N$, are the abscissas and weights of an $N$-point Gauss-Hermite integration formula [21, p. 924].

\section{APPENDIX B}

In common OOK receivers, the received optical signal is filtered by a bandpass optical filter, photodetected, and filtered again by a lowpass postdetection filter. The pdf of the current after the postdetection filter can be exactly evaluated by using a Karhunen-Loève series expansion method [22]-[25]. In the following text, we will shortly review the procedure for BER or pdf evaluation, mainly following [23], where the noise and the signal are expanded on two different Fourier bases, and the pdf is obtained by inverting the moment generating function through the saddlepoint method. In particular, we will highlight the small modifications required to account for parametric gain. The extension to the non-AWGN case is done by following [26], the PSD matrix is evaluated by adapting the CRLP model to a modulated signal, and a slightly different form of the moment generating function is adopted.

In the presence of nonlinearities, the modulated noise-free signal at the input of the photodetector is evaluated by propagating the $2^{N}$-bit de Bruijn transmitted sequence through the whole system by the split-step Fourier method. For each received bit at sampling time $t_{k}, k=1, \ldots, 2^{N}$, a different output PSD matrix $\mathrm{G}_{k}(z, \omega)$ is evaluated as explained in Section III, considering a $\mathrm{CW}$ signal with power $P_{k}$ that is equal to the transmitted power at time $t_{k}$. Following the notation adopted in [23], the photodetected sample at $t_{k}$ for each bit can be expressed as

$$
y\left(t_{k}\right)=c+\mathbf{n}^{T *} \mathbf{v}+\mathbf{v}^{T *} \mathbf{n}+\mathbf{n}^{T *} \mathbf{Q} \mathbf{n}
$$

where $\mathbf{n}$ is a complex vector containing the most relevant $2 M+1$ Fourier components of the optical noise at the input of the photodetector, and $c$ is the noise-free sample. The complex vector $\mathbf{v}$ and the Hermitian matrix Q, defined in [23], account for signalnoise and noise-noise beating, respectively. Since parametric gain breaks the symmetry between the inphase and quadrature noise components, we rewrite (B1) as

$$
y\left(t_{k}\right)=c+\mathbf{n}_{d}^{T} \mathbf{v}_{d}+\mathbf{v}_{d}^{T} \mathbf{n}_{d}+\mathbf{n}_{d}^{T} \mathrm{Q}_{d} \mathbf{n}_{d}
$$

where

$$
\begin{aligned}
\mathbf{v}_{d} & =\left[\begin{array}{l}
\operatorname{Re}\left\{\exp \left(-j \phi_{k}\right) \mathbf{v}\right\} \\
\operatorname{Im}\left\{\exp \left(-j \phi_{k}\right) \mathbf{v}\right\}
\end{array}\right] \\
\mathbf{n}_{d} & =\left[\begin{array}{l}
\operatorname{Re}\left\{\exp \left(-j \phi_{k}\right) \mathbf{n}\right\} \\
\operatorname{Im}\left\{\exp \left(-j \phi_{k}\right) \mathbf{n}\right\}
\end{array}\right] \\
\mathrm{Q}_{d} & =\left[\begin{array}{ll}
\operatorname{Re}\{\mathrm{Q}\} & -\operatorname{Im}\{\mathrm{Q}\} \\
\operatorname{Im}\{\mathrm{Q}\} & \operatorname{Re}\{\mathrm{Q}\}
\end{array}\right]
\end{aligned}
$$

and $\phi_{k}$ is the phase of the optical sample at the output of the fiber at time $t_{k}$, which is needed to account for the proper phase relationship between the noise and signal. The covariance matrix of $\mathbf{n}_{d}$, obtained directly from [23], is not diagonal. However, the vector $\mathbf{n}_{d}$ can be related to another noise vector $\mathbf{w}_{d}$ through a whitening operation $\mathbf{n}_{d}=T \mathbf{w}_{d}$, so that $\mathbf{w}_{d}$ is a zero mean Gaussian vector with diagonal covariance matrix $E\left\{\mathbf{w}_{d} \mathbf{w}_{d}^{T}\right\}=$ $\sigma^{2}$ I, where $\sigma^{2}=N_{0} /\left(2 T_{0}\right), T_{0}$ is the duration of the overall impulse response of the system defined in [23], and I is the identity matrix. We note that in the presence of parametric gain, that is not an instantaneous effect, the convergence of the expansions in (B1) or (B2) can be reached for a larger value of $T_{0}$ than in [23]. The matrix $T$ can be derived from the output noise PSD matrix $\mathrm{G}_{k}(z, \omega)$. Hence, we assume that the impulse response of the optical filter is real and its bandwidth is larger than $\phi$, so that we can neglect the phase-to-amplitude conversion caused by the optical filter [19]. Therefore, after photodetection, the phase term $\phi$ of the CRLP model in (5) disappears and only the PSD and cross-PSD of $a$ and $b$ are needed. The matrix T can be 
evaluated as in [26], $\mathrm{T}=\mathrm{BHB}^{-1}$, where $\mathrm{H}$ is a block matrix derived from the Cholesky decomposition of $\mathrm{G}_{k}(z, \omega)$

$$
\begin{aligned}
& \mathbf{H}=\left[\begin{array}{cc}
\operatorname{diag}\left\{h_{1,1}\left(\frac{m}{T_{0}}\right)\right\} & 0 \\
\operatorname{diag}\left\{h_{2,1}\left(\frac{m}{T_{0}}\right)\right\} & \operatorname{diag}\left\{h_{2,2}\left(\frac{m}{T_{0}}\right)\right\}
\end{array}\right] \\
& h_{1,1}(\omega)=\sqrt{G_{a a}(\omega)} \\
& h_{2,1}(\omega)=\frac{G_{a b}^{*}(\omega)}{\sqrt{G_{a a}(\omega)}} \\
& h_{2,2}(\omega)=\sqrt{\left|\frac{G_{b b}(\omega)-\left|G_{a b}(\omega)\right|^{2}}{G_{a a}(\omega)}\right|}
\end{aligned}
$$

and $B$ is a block matrix which relates the inphase/quadrature basis (adopted for $G_{k}$ ) to the real/imaginary basis (adopted for $\mathbf{n}_{d}$ and $\left.\mathbf{w}_{d}\right)$

$$
\mathrm{B}=\mathrm{B}^{-1}=\frac{1}{2}\left[\begin{array}{cc}
\mathrm{I}+\mathrm{D} & j(\mathrm{I}-\mathrm{D}) \\
-j(\mathrm{I}-\mathrm{D}) & \mathrm{I}+\mathrm{D}
\end{array}\right] .
$$

In (B5), I is the identity matrix and D is an antidiagonal matrix with all of the antidiagonal elements being equal to one. After the whitening operation, the problem is reduced, as in [23], to evaluate the pdf of the sum of noncentral chi-square independent random variables plus some constant terms. After some calculations and the diagonalization of the matrix $A=T^{T} Q_{d} T$, it can be shown that the moment generating function of $y\left(t_{k}\right)$ can be written as

$$
\Psi_{y_{k}}(s)=\exp (c s) \prod_{i=1}^{4 M+2} \frac{1}{\sqrt{1-\lambda_{i} \sigma^{2} s}} \exp \left(\frac{\sigma^{2}\left|b_{i}\right|^{2} s^{2}}{1-\lambda_{i} \sigma^{2} s}\right)
$$

where $b_{i}, i=1, \ldots, 4 M+2$ are the elements of the vector $\mathbf{b}=\mathrm{U}^{T} \mathrm{~T}^{T} \mathbf{v}_{d}, \mathrm{U}$ is the matrix of the eigenvectors of $\mathrm{A}$, and $\lambda_{i}, i=1, \ldots, 4 M+2$ are the corresponding eigenvalues. The expression in (B6) is slightly different from the one reported in [23], where the contribution of the constant terms was separated. Although theoretically equivalent, the straight use of (B6) avoids the singularity that arises in the expression reported in [23] when one or more eigenvalues are equal to zero [27]. Finally, the pdf can be evaluated by a saddle-point integration of $\Psi_{y_{k}}(s)$ (or $\Psi_{y_{k}}(s) / s$ for the BER), and by averaging all bits of the sequence $k=1, \ldots, 2^{N}$, exactly as described in [23]. In the special case of a true $\mathrm{CW}$ signal, which is considered in Section V-B, the described procedure is significantly simplified, since the signal propagation and expansion become trivial, and sequence averaging is not required.

\section{ACKNOWLEDGMENT}

The authors would like to thank J. Zweck, B. Marks, and O. Sinkin for fruitful and stimulating discussions.

\section{REFERENCES}

[1] G. P. Agrawal, Nonlinear Fiber Optics. London, U.K.: Academic Press, 1995.

[2] O. V. Sinkin, R. Holzlöhner, J. Zweck, and C. R. Menyuk, "Optimization of the split-step Fourier method in modeling optical-fiber communications systems," J. Lightw. Technol., vol. 21, no. 1, pp. 61-68, Jan. 2003.

[3] M. Karlsson, "Modulational instability in lossy optical fibers," J. Opt. Soc. Amer. B, vol. 12, no. 11, pp. 2071-2077, Nov. 1995.
[4] A. V. T. Cartaxo, "Small-signal analysis for nonlinear and dispersive optical fibres, and its application to design of dispersion supported transmission systems with optical dispersion compensation," Proc. Inst. Elect. Eng., Optoelectron., vol. 146, no. 5, pp. 213-222, Oct. 1999.

[5] A. Vannucci, P. Serena, and A. Bononi, "The RP method: A new tool for the iterative solution of the nonlinear Schrödinger equation," $J$. Lightw. Technol., vol. 20, no. 7, pp. 1102-1112, Jul. 2002.

[6] E. Ciaramella and E. Forestieri, "Analytical approximation of nonlinear distortions," IEEE Photon. Technol. Lett., vol. 17, no. 1, pp. 91-93, Jan. 2005.

[7] E. Forestieri and M. Secondini, "Solving the nonlinear Schrödinger equation," in Optical Communication Theory and Techniques. New York: Springer, 2004, pp. 3-11.

[8] R. Hui, M. O'Sullivan, A. Robinson, and M. Taylor, "Modulation instability and its impact in multispan optical amplified IMDD systems: Theory and experiments," J. Lightw. Technol., vol. 15, no. 7, pp. 1071-1082, Jul. 1997.

[9] A. Carena, V. Curri, R. Gaudino, P. Poggiolini, and S. Benedetto, "New analytical results on fiber parametric gain and its effects on ASE noise," IEEE Photon. Technol. Lett., vol. 9, no. 4, pp. 535-537, Apr. 1997.

[10] R. Holzlöhner, V. S. Grigoryan, C. R. Menyuk, and L. a. Kath, "Accurate calculation of eye diagrams and bit error rates in optical transmission systems using linearization," J. Lightw. Technol., vol. 20, no. 3, pp. 389-400, Mar. 2002.

[11] P. Serena, A. Bononi, J.-C. Antona, and S. Bigo, "Parametric gain in the strongly nonlinear regime and its impact on $10-\mathrm{Gb} / \mathrm{s} \mathrm{NRZ} \mathrm{systems}$ with forward-error correction," J. Lightw. Technol., vol. 23, no. 6, pp. 2352-2363, Aug. 2005.

[12] M. Secondini, E. Forestieri, and C. R. Menyuk, "A novel perturbation method for signal-noise interaction in nonlinear dispersive fibers," presented at the OFC, Anaheim, CA, Mar. 2006, paper OThD3.

[13] J. P. Gordon and L. F. Mollenauer, "Phase noise in photonic communications systems using linear amplifiers," Opt. Lett., vol. 15, no. 23, pp. 1351-1353, Dec. 1990.

[14] A. Mecozzi, "Error probability of amplified IMDD systems at zero dispersion," Electron. Lett., vol. 29, no. 24, pp. 2136-2137, Nov. 1993.

[15] K.-P. Ho, "Impact of nonlinear phase noise to DPSK signals: A comparison of different models," IEEE Photon. Technol. Lett, vol. 16, no. 5, pp. 1403-1405, May 2004.

[16] B. A. Berg, "Algorithmic aspects of multicanonical simulations," Nucl. Phys. B (Proc. Suppl.), vol. 63A-C, pp. 982-984, Apr. 1998.

[17] R. Holzlöhner and C. R. Menyuk, "Use of multicanonical monte carlo simulations to obtain accurate bit error rates in optical communications systems," Opt. Lett., vol. 28, pp. 1894-1896, Oct. 2003.

[18] L. Gerardi, M. Secondini, and E. Forestieri, "Pattern perturbation method for multicanonical Monte Carlo simulations in optical communications," IEEE Photon. Technol. Lett., vol. 19, no. 23, pp. 1934-1936, Dec. 2007.

[19] E. Forestieri, "PM/AM and AM/PM conversions in linear optical fibers," in Opt. Netw., A. Bononi, Ed. Berlin, Germany: Springer-Verlag, 1999 , pp. 364-382.

[20] A. Papoulis, Probability, Random Variables, and Stochastic Processes, 3rd ed. New York: McGraw-Hill, 1991.

[21] M. Abramowitz and I. A. Stegun, Handbook of Mathematical Functions. New York: Dover, 1972

[22] M. Kac and A. J. F. Siegert, "On the theory of noise in radio receivers with square law detectors," J. Appl. Phys., vol. 18, no. 4, pp. 383-397, Apr. 1947.

[23] E. Forestieri, "Evaluating the error probability in lightwave systems with chromatic dispersion, arbitrary pulse shape and pre- and postdetection filtering," J. Lightw. Technol., vol. 18, no. 11, pp. 1493-1503, Nov. 2000.

[24] J.-S. Lee and C.-S. Shim, "Bit-error-rate analysis of optically preamplified receivers using an eigenfunction expansion method in optical frequency domain," J. Lightw. Technol., vol. 12, no. 7, pp. 1224-1229, Jul. 1994.

[25] G. Bosco, A. Carena, V. Curri, R. Gaudino, P. Poggiolini, and S. Benedetto, "A novel analytical approach to the evaluation of the impact of fiber parametric gain on the bit error rate," IEEE Trans. Commun., vol. 49, no. 12, pp. 2154-2163, Dec. 2001.

[26] P. Serena, A. Orlandini, and A. Bononi, "Parametric gain approach to the analysis of single-channel DPSK/DQPSK systems with nonlinear phase noise," J. Lightw. Technol., vol. 24, no. 5, pp. 2026-2037, May 2006.

[27] E. Forestieri and M. Secondini, "On the error probability evaluation in lightwave systems with optical amplification,” J. Lightw. Technol., vol. 27, no. 6, pp. 706-717, Mar. 15, 2009. 


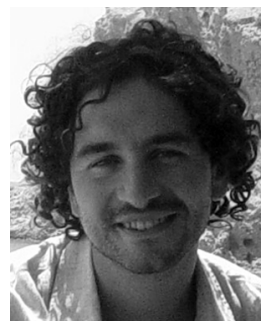

Marco Secondini was born in Rome, Italy, in 1975. He received the Dr.Ing. degree in electronics engineering from the University of Roma Tre, Rome, Italy, in 2000, and the Ph.D. degree from Scuola Superiore Sant'Anna, Pisa, Italy, in 2006.

During 2001, he was with QPlus Networks, Inc, developing fiber-optic systems for ultra long-haul communications. Since 2002, he has been with the Scuola Superiore Sant'Anna, Pisa, where he is currently an Assistant Professor. He also collaborates with the National Photonic Networks Laboratory of the CNIT in Pisa. During 2005, he was a Visiting Faculty Research Assistant in the Photonics Group, University of Maryland, Baltimore County, Baltimore. His current research interests are in the area of optical communication theory and include fiber nonlinearities, chromatic- and polarization-mode dispersion, optical equalization techniques, and Monte Carlo methods for simulations.

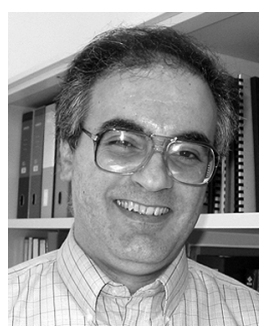

Enrico Forestieri (S'91-M'92) was born in Milazzo, Italy, in 1960. He received the Dr.Ing. degree in electronics engineering from the University of Pisa, Pisa, Italy, in 1988.

From 1989 to 1991, he was a Postdoctoral Scholar at the University of Parma, Parma, Italy, working on optical-communication systems. From 1991 to 2000, he was a Research Scientist and Faculty Member with the University of Parma. Since 2001, he has been with Scuola Superiore Sant'Anna di Studi Universitari e di Perfezionamento, Pisa, where he is currently Professor of Telecommunications. His research interests are in the general area of digital communication theory and optical-communication systems, with special attention to adaptive optical and electronic equalization, channel coding, and advanced modulation formats for optical systems. He is the leader of the "Optical Transmission Theory and Techniques" area at the Integrated Research Center for Photonic Networks and Technologies (IRCPhoNeT), Pisa. His research activity has led to numerous scientific publications in leading international journals and conference proceedings as well as a few patents.

Dr. Forestieri was General Chairman of the Tyrrhenian International Workshop on Digital Communications in 2004.

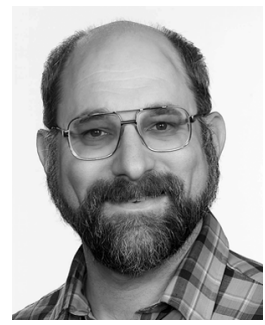

Curtis R. Menyuk (F'98) was born March 26, 1954. He received the B.S. and M.S. degrees from the Massachusetts Institute of Technology (MIT), Cambridge, in 1976 and the Ph.D. degree from the University of California at Los Angeles (UCLA) in 1981.

He was a Research Associate at the University of Maryland, College Park, and at Science Applications International Corporation, McLean, VA. In 1986, he became an Associate Professor in the Department of Electrical Engineering at the University of Maryland, Baltimore County (UMBC), Baltimore, and was the founding member of this department. In 1993, he was promoted to Professor. He was on partial leave from UMBC from 1996 until 2002. From 1996 to 2001, he worked part-time for the Department of Defense, codirecting the Optical Networking program at the DoD Laboratory for Telecommunications Sciences, Adelphi, MD, from 1999 to 2001. From 2001 to 2002, he was Chief Scientist at PhotonEx Corporation. For the last 20 years, his primary research area has been theoretical and computational studies of lasers, nonlinear optics, and fiber-optic communications. He has authored or coauthored more than 210 archival journal publications as well as many other publications and presentations. He has also edited three books. The equations and algorithms that he and his research group at UMBC have developed to model optical-fiber systems are used extensively in the telecommunications and photonics industry.

Dr. Menyuk is a member of the Society for Industrial and Applied Mathematics. He is a Fellow of the American Physical Society and the Optical Society of America. He is a former UMBC Presidential Research Professor. 